\title{
Addendum to Nonperturbative QED: Muon Structure and Decay
}

\author{
Burke Ritchie $^{1}$ \\ ${ }^{1}$ Lawrence Livermore National Laboratory, Livermore, CA 94550, USA \\ Correspondence: Burke Ritchie, Lawrence Livermore National Laboratory, Livermore, CA 94550, USA. E-mail: \\ ritchie@1stc.com
}

Received: November 5, 2014 Accepted: November 11, 2014 Online Published: November 26, 2014

doi:10.5539/apr.v6n6p103

URL: http://dx.doi.org/10.5539/apr.v6n6p103

\begin{abstract}
In previous work the mutual binding of an electron and two neutrinos was studied in which the electron was treated dynamically. In this addendum it is shown that neutrinos can bind to an electron at rest. This picture gives a simpler and more physically realistic model for the muon. For completeness a fresh presentation of the neutrino equation of motion is given.
\end{abstract}

Keywords: muon, neutrino, electron, mass-energy equivalence

In previous work on the structure and decay of the muon (Ritchie, 2014) the electron was treated dynamically. In this paper I describe a simpler and more physically realistic model in which neutrinos are bound to an electron at rest. The transient mediating particle for the electroweak force, the $\mathrm{W}^{-b}$ boson, decays into an electron and neutrino. In absence of a dynamically resolution of the force-carrier motion it nevertheless should be possible to infer the physical effects of the electroweak interaction net of force-carrier dynamics by finding a physically appropriate equation of motion (EOM) for the electron-neutrino.

In analogy to Dirac's equation for the electron's material aspect given in Ritchie (2014), I infer a neutrino EOM from the Lorentz invariant found from the scalar product of a renormalized four-gradient and a second electromagnetic 4-potential which I postulate to account for the electron's intrinsic electromagnetic properties as measured by its charge,

$$
\left(\frac{1}{c} \frac{\partial}{\partial t}, \vec{\nabla} \pm \frac{e}{m c^{2}} \vec{E}, \vec{H}\right) \cdot\left(\Phi_{v}, \vec{A}_{v}\right)=\frac{1}{c} \frac{\partial}{\partial t} \Phi_{e}+\left(\hbar \vec{\nabla} \pm \frac{e}{m c^{2}} \vec{E}, \vec{H}\right) \cdot \vec{A}_{v}=0,
$$

where the upper (lower) sign stands for neutrino (antineutrino) and the notation $\vec{E}, \vec{H}$ means electric or magnetic field respectively which is understood to be external to the neutrino due to the presence of an electron. (The subscripts denoting interactions external to an electron or neutrino are henceforth dropped.) The scalar product of

the renormalized 4-gradient, $\left(\frac{1}{c} \frac{\partial}{\partial t}, \vec{\nabla} \pm \frac{e}{m c^{2}} \vec{E}, \vec{H}\right)$, and the electromagnetic 4-current, $\left(c\left(u+\int_{0}^{t} d t \vec{j} \cdot \vec{E}\right), \vec{S}\right)$, gives the electromagnetic equation of continuity,

$$
\frac{\partial u}{\partial t}+\vec{\nabla} \cdot \vec{S}+\vec{j} \cdot \vec{E}=0
$$

since the scalar product of the $\vec{E}$ or $\vec{H}$ with $\vec{S}$ vanishes, where $u=\frac{1}{8 \pi}\left(E^{2}+H^{2}\right)$ is the electromagnetic energy density and $\vec{S}=\frac{c}{4 \pi} \vec{E} \times \vec{H}$ is the electromagnetic 3-current. Dirac required his equation for a relativistic electron to be compatible with the material equation of continuity. Thus I believe that a complete description of a relativistic electron, in which its radiant aspect responsible for its Lamb shift and its anomalous magnetic moment are properly understood without needing to remove infinite contributions using renormalization schemes, should also comprise equations of motion which are compatible with the electromagnetic equation of continuity given by Equation (2). The posited 4-potential given in Equation (1) can be written in the form of carrier-wave expansions, 


$$
\begin{gathered}
\Phi_{v}=\Phi_{v_{-}} e^{-i \omega_{v} t}+\Phi_{v_{+}} e^{i \omega_{v} t} \\
\vec{A}_{v}=\vec{A}_{v-} e^{-i \omega_{v} t}+\vec{A}_{v+} e^{i \omega_{v} t},
\end{gathered}
$$

from which on substituting Equations (3) into Equation (1) and separately setting the coefficients of the exponential factors equal to zero, we obtain,

$$
\begin{aligned}
& \left(\frac{1}{c} \frac{\partial}{\partial t}+i \frac{\omega_{v}}{c}\right) \Phi_{v+}+\left(\vec{\nabla} \pm \frac{e}{m c^{2}} \vec{E}, \vec{H}\right) \cdot \vec{A}_{v+}=0 \\
& \left(\frac{1}{c} \frac{\partial}{\partial t}-i \frac{\omega_{v}}{c}\right) \Phi_{v-}+\left(\vec{\nabla} \pm \frac{e}{m c^{2}} \vec{E}, \vec{H}\right) \cdot \vec{A}_{v-}=0 .
\end{aligned}
$$

On setting $\Phi_{v+}=\xi_{E, H}, \vec{A}_{v+}=\vec{\sigma} \zeta_{E, H}, \Phi_{v_{-}}=\zeta_{E, H}, \vec{A}_{v-}=\vec{\sigma} \xi_{E, H}$ we obtain the Dirac form postulated for the neutrino,

$$
\begin{aligned}
& \frac{\partial \xi_{E, H}}{c \partial t}+i \frac{\omega_{v}}{c} \xi_{E, H}+\vec{\sigma} \cdot\left(\vec{\nabla} \pm \frac{e}{m c^{2}} \vec{E}, \vec{H}\right) \zeta_{E, H}=0 \\
& \frac{\partial \zeta_{E, H}}{c \partial t}-i \frac{\omega_{v}}{c} \zeta_{E, H}+\vec{\sigma} \cdot\left(\vec{\nabla} \pm \frac{e}{m c^{2}} \vec{E}, \vec{H}\right) \xi_{E, H}=0 .
\end{aligned}
$$

Writing $\xi_{E, H}=e^{-i \omega t} \psi_{E, H}$ and $\zeta_{E, H}=e^{-i \omega t} \chi_{E, H}$ in Equations (5) we derive stationary equations for $\psi_{E, H}$ and $\chi_{E, H}$; then we eliminate the equation for $\chi_{E, H}$ in favor of a second-order equation for $\psi_{E, H}$,obtaining equations for the electric and magnetic neutrino wave functions which have the Helmholtz form,

$$
\begin{gathered}
\left\{\nabla^{2}+\frac{\omega^{2}-\omega_{v}^{2}}{c^{2}} \pm \frac{e}{m c^{2}}\left[\vec{\nabla} \cdot \vec{E}+2 \vec{E} \cdot \vec{\nabla}+i \sigma \cdot(\vec{\nabla} \times \vec{E}) \pm \frac{e}{m c^{2}} E^{2}\right]\right\} \psi_{E}=0 \\
\left\{\nabla^{2}+\frac{\omega^{2}-\omega_{v}^{2}}{c^{2}} \pm \frac{e}{m c^{2}}\left[\vec{\nabla} \cdot \vec{H}+2 \vec{H} \cdot \vec{\nabla}+i \sigma \cdot(\vec{\nabla} \times \vec{H}) \pm \frac{e}{m c^{2}} H^{2}\right]\right\} \psi_{H}=0,
\end{gathered}
$$

where we have used the identity, $(\vec{\sigma} \cdot \vec{A})(\vec{\sigma} \cdot \vec{B})=\vec{A} \cdot \vec{B}+i \vec{\sigma} \cdot(\vec{A} \times \vec{B})$.

Notice that in Equations (4)-(6) external electromagnetic fields and not external electromagnetic potentials occur such that there is no question of a gauge dependence of neutrino-electron interactions in the neutrino EOM. As shown below in the electric-field and magnetic-field equations of motion with second-order Helmholtz form

[Equations (6)], the same-parity, $\vec{\nabla} \rightarrow \vec{\nabla} \pm \frac{e}{m c^{2}} \vec{E}$, and mixed-parity, $\vec{\nabla} \rightarrow \vec{\nabla} \pm \frac{e}{m c^{2}} \vec{H}$, addition vectors contribute, among other terms, all four of Maxwell's equations as interaction terms, the same parity addition vector contributing $\vec{\nabla} \cdot \vec{E}=4 \pi e \rho$ and $\vec{\nabla} \times \vec{E}=-\frac{1}{c} \frac{\partial \vec{H}}{\partial t}$ and the mixed parity, addition vector contributing $\vec{\nabla} \cdot \vec{H}=0$ and $\vec{\nabla} \times \vec{H}=\frac{4 \pi e}{c} \vec{j}+\frac{1}{c} \frac{\partial \vec{E}}{\partial t}$.

I consider the binding of two neutrinos with an electron at rest. The EOM's for the neutrino, as discussed in the previous section, is given by Equation (6a) as follows,

$$
\text { neutrino EOM: } E_{v}^{2} \psi_{E}+\hbar^{2} c^{2}\left\{\nabla^{2}+\frac{e}{m c^{2}}\left[\vec{\nabla} \cdot \vec{E}+2 \vec{E} \cdot \vec{\nabla}+\frac{e}{m c^{2}} E^{2}\right]\right\} \boldsymbol{\psi}_{E}=0
$$

where in Equation (6a) $\omega_{v}=\frac{m_{v} c^{2}}{\hbar}=0 \quad$ (neutrino assumed to have zero mass), $\omega=\frac{E_{v}}{\hbar}$, and I have ignored the spin-dependent dynamical contribution.

The interaction of the neutrino with the electron in the neutrino EOM is the electric field arising from the charge density of the electron, $e \vec{E}=-\vec{\nabla} V$,

$$
V=e^{2}\left[\frac{1}{r} \int_{0}^{r} d r^{\prime} r^{\prime 2}\left(G_{-1}^{2}+F_{-1}^{2}\right)+\int_{r}^{\infty} d r^{\prime} r^{\prime}\left(G_{-1}^{2}+F_{-1}^{2}\right)\right]
$$


where the electronic density is inferred from Equation (7)using the large and small components of the wave function for one neutrino $\psi_{E}=G_{\kappa}(r) \chi_{\kappa \mu}(\theta, \phi), \chi_{E}=F_{\kappa}(r) \chi_{-\kappa \mu}(\theta, \phi)$ and similarly for the other neutrino using lower cases for the radial components. From the foregoing it is clear that the neutrino-electron interaction is physically equivalent to an electron's self-electromagnetic-field interaction and thus accounts for the electron's charge and self interaction which is absent in Dirac's theory of a relativistic electron, which obviously accounts only for the electron's spin and rest-mass energy.

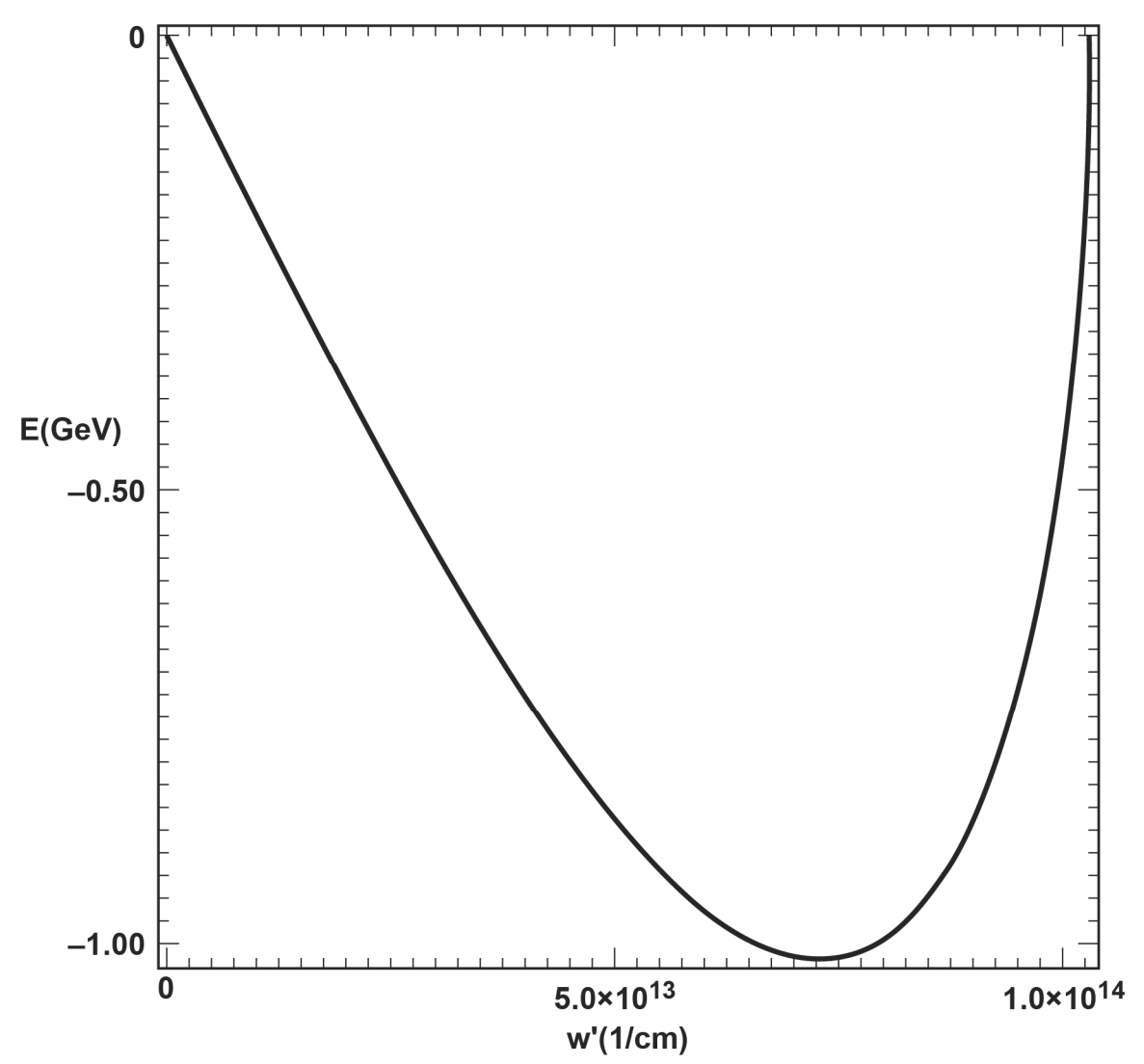

Figure 1. Muon energy versus variational parameter $W^{\prime}$ in the approximate solution of Equation (13)

Proceeding heuristically the radial equations (for $\kappa=-1$ ) are solved variationally using the unnormalized trial forms $G_{-1}(r)=e^{-W T}$ and $g_{-1}(r)=e^{-W^{\prime} r} h_{-1}(r)$ for the "large" components, where $W^{\prime}=2.9 W$. The "small" components are calculated using the trial forms, $f_{-1}(r)=\frac{\hbar c}{m_{\text {muо }} c^{2}} g_{-1}^{\prime}$ and $F_{-1}(r)=\frac{\hbar c}{m_{W} c^{2}} G_{-1}^{\prime}$, where experimental parameters are used for the muon and W-boson masses respectively. (Calculations show that the results are insensitive to these mass choices but are sensitive to the relative values of the variational parameters $\mathrm{w}$ and $W^{\prime}$.) 


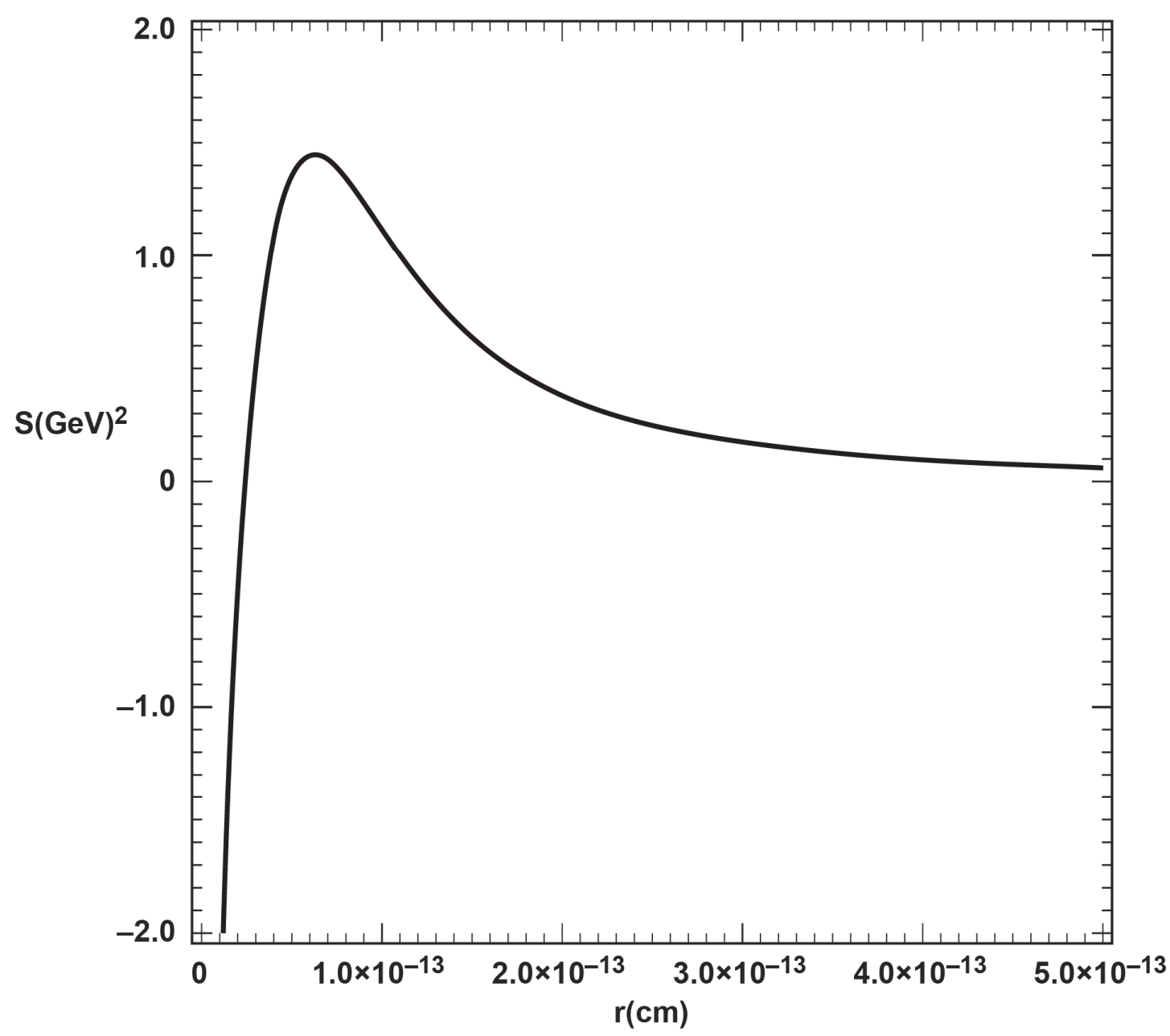

Figure 2. The "potential" function $\mathrm{S}(\mathrm{r})^{2}$ in Equations (15a) versus $\mathrm{r}$ which occurs for the $w^{\prime}$ value at energy-curve minimum given in Figure $1 . W^{\prime}=2.9 W$ where $W^{\prime}$ is the variational parameter used for the approximate muon solution of Equations (13) while $\mathrm{w}$ is the variational parameter used for the $\mathrm{W}^{-}$boson solution to the same equation, in which $w$ is used to calculate the electric field in Equations (13) for the $w^{\prime}$ solution and $W^{\prime}$ is used to calculate the electric field in Equations (13) for the w solution. The ratio of $w^{\prime}$ to $w$ is adjusted to to give the lifetime of $2.05 \times 10^{-6} \mathrm{~s}$, which is close to the experimentally observed lifetime of $2.20 \times 10^{-6} \mathrm{~s}$ assuming the experimentally observed positive energy behind the barrier of $100 \mathrm{MeV}$. A similar calculation for the lifetime of the posited $\mathrm{W}^{-}$boson against emission of an antineutrino gives $1.73 \times 10^{-23} \mathrm{~s}$ assuming an energy of $80 \mathrm{GeV}$

Once the derivative operation has been carried out on the trial function Equation (7) has the standard Schroedinger form where in Equation (6a) below the contribution given by $\frac{2 e \hbar^{2}}{m} \vec{E} \cdot \vec{\nabla} \psi_{E}$ is evaluated approximately by replacing it with $-\frac{2 e \hbar^{2} w^{\prime}}{m} \vec{E} \cdot \hat{r} \psi_{E}$ in which it is assumed that $w^{\prime}>>\left|\frac{d}{d r} h_{-1}(r)\right|$ Once the derivative operation indicated inthe interaction contribution, $\frac{2 e \hbar^{2}}{m} \vec{E} \cdot \vec{\nabla} \psi_{E}$, has been approximately evaluated as outlined above Equation (7) has the Schroedinger form,

$$
\left[E_{v}^{2}+\hbar^{2} c^{2} \nabla^{2}-S(r)^{2}\right] \psi_{E}=0,
$$




$$
S(r)^{2}=-\frac{e^{2} \hbar^{2}}{m}\left(\rho_{r}+\frac{V^{\prime}}{e^{2}} W^{\prime}+\frac{1}{m c^{2} e^{2}} V^{\prime 2}\right),
$$

where $S(r)^{2}$ is plotted versus $r$ in Figure (2). The first and third terms on the right side of Equation (15b) are attractive while the second term is repulsive such that a neutrino can bind to an electron or positron behind the squared potential barrier shown in Figure 2. Notice that the barrier in Figure 2 would be inverted for an antineutrino in this methodology such that, for zero mass, the bound energy would be imaginary. Notice also that the shape of the squared-energy curve with a region of strong attraction near the origin has the standard form of the strong force in nuclear theory. Standard WKB theory (Bethe \& Salpeter, 1977) can be used to estimate the energy levels behind the barrier and the tunneling rate through the barrier, whence

$$
I=\int_{r_{1}}^{r_{2}} d r \sqrt{\kappa_{v}^{2}-\frac{S(r)^{2}}{\hbar^{2} c^{2}}}=\left(n+\frac{1}{2}\right) \pi
$$

where $\kappa_{v}=\frac{E_{v}}{\hbar c}$ and the limits of integration are over the classical region in which the argument under the square-root sign is positive, and where the integration limits in the exponential term are over the barrier width for the value of $\mathrm{w}$ giving the minimum energy in Figure 2. An energy $E_{v}$ is chosen for which the reciprocal of the tunneling rate is nearly equal to the lifetime of the muon. The calculated rate is $4.88 \times 10^{5} \mathrm{~s}^{-1}$, whose reciprocal gives a lifetime of $2.05 \times 10^{-6} \mathrm{~s}$, which is close to the observed muon lifetime of nearly $2.20 \times 10^{-6} \mathrm{~s}$. This rate corresponds to $100 \mathrm{MeV}$ chosen for the muon rest-mass enery, which is taken to be the positive energy behind the squared energy barrier shown in Figure 2. In atomic units the numerator in Equation (11) is $2.42 \times 10^{-16}$ and the integral in the denominator is $5.13 \times 10^{-6}$, such that the quotient is $1.18 \times 10^{-11}$, which, converted to cgs units by division by the atomic unit of time, $2.42 \times 10^{-17}$, gives the the rate cited above. An antineutrino (see Equation (4) in which the lower sign is taken for the renormalized gradient) is emitted by a posited $\mathrm{W}^{-}$boson, which the same methodology used to calculate the lifetime of the neutrino discussed above gives a lifetime of $1.73 \times 10^{-23} \mathrm{~s}$ for an assumed $\mathrm{W}^{-}$boson energy of $80 \mathrm{GeV}$. The change of sign in the renormalized gradient causes the antineutrino squared energy curve to be an inverted version of the neutrino squared energy curve shown in Figure 2, such that no bound states exist in the positive-energy regime.

$$
R=\frac{\hbar}{m} \frac{e^{-2 \int_{r_{2}}^{r_{3}} d r \sqrt{\frac{S(r)^{2}}{\hbar^{2} c^{2}}-\kappa_{v}^{2}}}}{4 \int_{r_{1}}^{r_{2}} d r\left[\left(\kappa_{v}^{2}-\frac{S(r)^{2}}{\hbar^{2} c^{2}}\right]^{-\frac{1}{2}}\right.} \mathrm{s}^{-1},
$$

The experimentally observed weakness of the interaction of a neutrino with matter is explained here by the smallness of the inverse process of the tunneling of a free neutrino back through the potential barrier to penetrate into the core region of strong binding.

Finally I should comment here on the scaling of the interaction terms in the two coupled equations so that readers feel comfortable with the nuclear-scale binding found in these calculations. $\vec{E}=-\vec{\nabla} \Phi$ is the electric field, where

$\Phi=\frac{e}{r}$ is the Coulomb potential due to a bare positron or bare electron respectively. Notice that the term $\frac{e^{2} \hbar^{2}}{m^{2} c^{2}} E^{2}=\frac{e^{2} \hbar^{2}}{m^{2} c^{2}}(\vec{\nabla} \Phi)^{2}$ in Equation (6a), which is in units of squared energy, isalways attractive such that its conribution can support bound states of the neutrino EOM. The interaction scales according to the quantum expectation value ofthe attractive interaction term, $\frac{e^{2} \hbar^{2}}{m^{2} c^{2}}(\vec{\nabla} \Phi)^{2}$, as $\frac{e^{4} \hbar^{2} w^{4}}{m^{2} c^{2}}$. The attraction scaling a $\frac{e^{4} \hbar^{2} W^{4}}{m^{2} c^{2}}$ can overcome with increasing $\mathrm{w}$ the kinetic energy (first term in the above operator) scaling as $\hbar^{2} c^{2} w^{2}$. The 
square root of $\frac{e^{4} \hbar^{2} W^{4}}{m^{2} c^{2}}$ is an energy in the $\mathrm{GeV}$ regime for a variational parameter w which is the reciprocal of the proton's Compton wavelength $W=\frac{m_{p} c}{\hbar}$, as the reader may easily verify.

In conclusion I have formulated a nonperturbative, divergence-free QED which describes the permanent contribution of radiation to the structure of matter and as well shows nuclear-scale binding. The foundation of the new QED is based on my belief that relativistic electron theory is incomplete if it is compatible only with the material equation of continuity and accounts only for the electron's rest-mass energy and spin. A complete relativistic-electron theory should also be compatible with the electromagnetic equation of continuity such that in toto it accounts for the electron's rest-mass energy, spin, charge, and self electromagnetic energy. The theory presented here accounts for these properties by including an EOM which appears to be physically appropriate for a neutrino at large electron-neutrino separations. The observed weakness of a free neutrino's interaction with matter would be manifest here in the smallness of the rate of tunnelling of free neutrinos from right to left back through the squared potential energy barrier shown in Figure 2 and can easily be estimated from the uncertainty principle $\Delta E \Delta t \cong \hbar$, where $\Delta t$ is the right-to left back tunnelling rate theough the potential barrier and $\Delta E$ is an estimate of the strength of the interaction between free neutrinos and matter. Future work should focus on the exact solution of Equation (13) to eliminate a dependence on empirical data.

\section{Acknowledgements}

The author is grateful to T. Scott Carman for supporting this work. He is grateful to Professor John Knoblock of the University of Miami for seminal discussion. This work was performed under the auspices of the Lawrence Livermore National Security, LLC, (LLNS) under Contract No. DE-AC52-07NA27344.

\section{Reference}

Ritchie, B. (2014). Nonperturbative QED: Muon Structure and Decay. Applied Physics Research, 6(5), 57-64.

Bethe, H. A., \& Salpeter, E. E. (1977). Quantum mechanics of one-and two-electron atoms (Vol. 168). New York: Plenum Publishing Corporation.

\section{Copyrights}

Copyright for this article is retained by the author(s), with first publication rights granted to the journal.

This is an open-access article distributed under the terms and conditions of the Creative Commons Attribution license (http://creativecommons.org/licenses/by/3.0/). 